\title{
The pocket-creation method of endoscopic submucosal dissection
}

\author{
Yoshikazu Hayashi', Yoshimasa Miura', Alan Kawarai Lefor ${ }^{2}$, Hironori Yamamoto' \\ 'Department of Medicine, Division of Gastroenterology, Jichi Medical University, Shimotsuke 329-0498, Japan. \\ ${ }^{2}$ Department of Surgery, Jichi Medical University, Shimotsuke 329-0498, Japan.
}

Correspondence to: Prof. Hironori Yamamoto, Department of Medicine, Division of Gastroenterology, Jichi Medical University, Shimotsuke 329-0498, Japan. E-mail: ireef@jichi.ac.jp

How to cite this article: Hayashi Y, Miura Y, Lefor AK, Yamamoto $\mathrm{H}$. The pocket-creation method of endoscopic submucosal dissection. Mini-invasive Surg 2022;6:7. https://dx.doi.org/10.20517/2574-1225.2021.125

Received: 30 Oct 2021 First Decision: 29 Dec 2021 Revised: 7 Jan 2021 Accepted: 13 Jan 2021 Published: 8 Feb 2022

Academic Editors: Giulio Belli, Shinji Tanaka Copy Editor: Xi-Jun Chen Production Editor: Xi-Jun Chen

\begin{abstract}
Endoscopic submucosal dissection (ESD) is rapidly becoming the standard treatment for superficial gastrointestinal tumors because ESD can achieve complete local resection facilitating thorough pathological examination of the resected specimen. The pocket-creation method (PCM) has been established to perform safe and reliable ESD obtaining a high-quality pathological specimen. A minimal mucosal incision using PCM minimizes leakage of submucosally injected solution, which results in prolonged mucosal elevation. A limited-space submucosal pocket created using PCM makes the endoscope tip stable. A conical cap, small-caliber-tip transparent (ST) hood is used when performing PCM. The submucosa can be cut along the ideal dissection line just above the muscularis with minimal thermal damage because the tip of the ST hood produces both traction and countertraction to stretch the submucosal tissue in the pocket. PCM is recommended as the standard strategy not only for colorectal ESD but also for upper-gastrointestinal ESD. It is expected that the use of traction techniques will make PCM easier to perform.
\end{abstract}

Keywords: Pocket-creation method, endoscopic submucosal dissection, ESD, colon, rectum, stomach, duodenum

\section{INTRODUCTION}

Endoscopic treatments have gradually been preferred over surgical resection because of their less invasiveness and their ability to preserve the original organs. In general, endoscopic treatments are 
indicated for superficial gastrointestinal tumors. Some submucosal invasive cancers have a risk for lymph node metastases. Endoscopic treatment can achieve local tumor resection without the eradication of regional lymph nodes. However, the risk can be predicted through pathological examination of resected specimens because the risk factors are present in the submucosa of the specimens. Therefore, when endoscopically resecting the tumors not only an Ro resection, which means resection of a specimen including the whole tumor with a negative margin, but also the less-damaged thick submucosa of the specimen is required to predict the risk of lymph node metastases.

Endoscopic submucosal dissection (ESD) is one type of endoscopic treatment that is becoming the standard treatment for superficial gastrointestinal tumors in the case of lesions especially challenging for conventional endoscopic resection (e.g., endoscopic polypectomy, endoscopic mucosal resection, etc.) because ESD can achieve an Ro resection with sufficient submucosa. However, ESD is still technically challenging for many endoscopists who have not had sufficient experience with the procedure. There are several factors for these difficulties. For instance, endoscopic maneuverability, thickness of the muscularis, the presence of blood vessels, degree of submucosal fibrosis, and fluctuations due to breathing and heartbeat differ in each organ, and even at each location within the organs.

Recently, the pocket-creation method (PCM) was developed to standardize the strategy of ESD. Efficacy of PCM was revealed not only in the colorectum but also in upper gastrointestinal tract ${ }^{[1-8]}$.

\section{The pocket-creation method}

PCM was originally developed to completely resect colorectal sub-pedunculated tumors with severe submucosal fibrosis ${ }^{[1]}$. Formerly, we performed ESD using the conventional strategy in which a mucosal incision was made for at least one-quarter of the circumference and submucosal dissection below the incised area were made alternatively ${ }^{[5]}$. However, when dissecting a tumor with severe submucosal fibrosis using the conventional strategy, it can be too difficult to identify and resect the fibrotic submucosa between the tumor and muscularis because the injected solution can leak out of the wide mucosal incision and the tip of the endoscope can be unstable in the opened submucosal space. Meanwhile, PCM with a small initial mucosal incision minimizes leakage of the injected solution and makes the endoscope tip stable in the submucosal pocket [Figure 1]. In addition, a conical transparent cap ${ }^{[9]}$, small-caliber-tip transparent (ST) hood (DH-33GR, DH-34CR, Fujifilm, Tokyo, Japan) naturally produces both traction and countertraction to stretch the fibrotic submucosa in the limited-space pocket, which facilitates cutting the submucosa. It is like stretching cloth when cutting it with scissors. Although PCM was initially developed to overcome challenging sessile colorectal tumors, it has been found to be useful in dissecting ordinary flat tumors such as laterally spreading tumors ${ }^{[2,5]}$. PCM has been the standard strategy for colorectal ESD, especially in our institution for a long time.

\section{Devices for PCM}

The ST hood is a key device for performing PCM. The ST hood facilitates entry of the tip of the endoscope to the submucosal layer quickly and produces traction from the center to the outside in the endoscopic view. A guide rail prepared inside the ST hood can also move the tip of the endoscopic device to the center of the endoscopic view. A needle-type knife such as Flushknife (Fujifilm) or Dualknife (Olympus, Tokyo, Japan) is appropriate for PCM because the centered tip of the needle-type knife cuts submucosal tissue in any direction. Injecting a viscous solution, such as $0.4 \%$ sodium hyaluronate, prolongs thickening of the submucosa which also facilitates $\mathrm{PCM}^{[9]}$. 

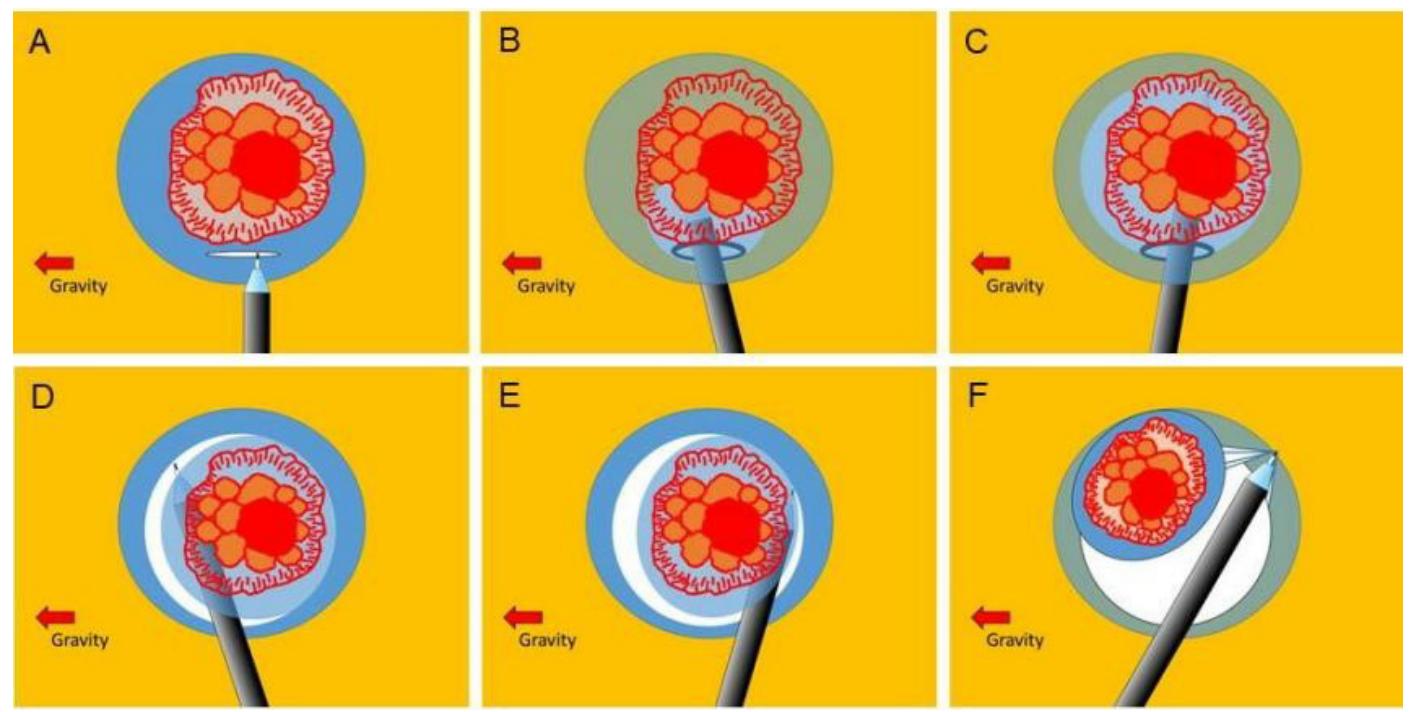

Figure 1. Sequence for the pocket-creation method (PCM) of endoscopic submucosal dissection (ESD). (A) An initial mucosal incision is made away from the edge of the tumor after submucosal injection. $(B, C)$ Creation of a submucosal pocket dissecting the submucosal tissue under the tumor. (D) Opening the pocket from the gravity side, up to beyond the tumor. (E) Dissection of the side opposite gravity in the same manner. (F) Completion of the ESD.

\section{Advantages of PCM}

Advantages of PCM include: (1) maintaining a thick submucosal layer because the minimal mucosal incision prevents leakage of injected solution; (2) the endoscopic view facilitates recognition of the surface of the muscularis because ST-hood traction in the narrow submucosal pocket stretches the submucosal tissue to identify the correct dissection line just above the muscularis; (3) obtaining a high-quality pathological specimen with a thick, less-damaged submucosal layer under the tumor; (4) adjusting the orientation in the pocket to make the tip of the endoscope horizontal to the muscularis; and (5) synchronization of the endoscope tip with fluctuations of the heartbeat and breathing in the pocket results in visual stability.

\section{Tips for performing PCM}

Some tips facilitate PCM in practice. Some of them are surely useful for not only PCM but also most ESDs.

\section{Aspirate the gas}

Luminal insufflation is required to obtain a good endoscopic view in the gastrointestinal tract. However, excessive insufflation interferes with endoscopic maneuverability. With PCM, however, luminal gas is not necessary because the working space is the submucosal layer, and the endoscopic view is maintained by the ST hood. Therefore, in PCM, good endoscopic control can be obtained by aspirating the luminal gas. Collapsing the intestine by aspirating the gas in it makes the intestinal wall tangential [Figure 2]. Even a vertical orientation can be changed to horizontal after aspirating the gas. Aspirating the gas keeps the submucosa thicker and the endoscope tip more stable in the narrowed lumen [Figure 3].

\section{The entrance should be distant from the tumor edge}

The initial mucosal incision should be made distant from the tumor edge to achieve greater traction and prevent mucosal-edge rolling. The specimen edge receives strong upward traction from the tip of the ST hood upon entering the submucosal space. A shorter tumor-side edge undergoes little upward traction from the tip of the ST hood. If the shorter edge is accidentally torn by traction, the tear will easily reach and 


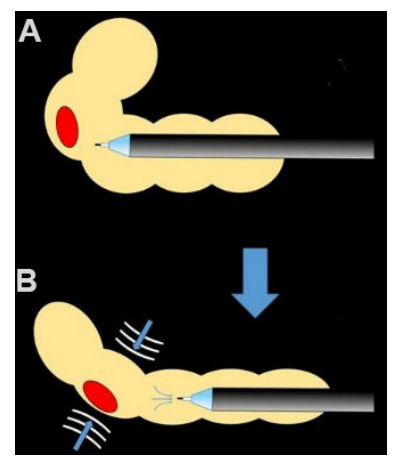

Figure 2. Changing the vertical orientation to horizontal after aspirating the gas. (A) A tumor is on the vertical wall. (B) The wall becomes horizontal after collapsing the intestine by aspirating gas.

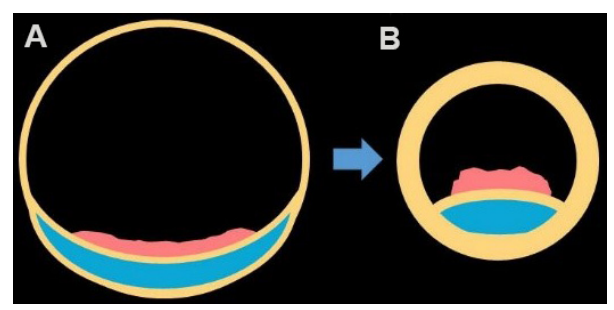

Figure 3. The submucosa thickens after aspirating the gas. (A) The submucosa is stretched and thinned in the insufflated intestine. (B) The submucosa thickens in the collapsed intestine.

damage the tumor. However, a longer tumor-side edge allows for greater traction [Figure 4]. Then, the endoscope goes downward gradually to reach the bottom of the submucosal layer. Even if the longer edge is accidentally torn by traction, it will have a margin at a distance. Coagulation when dissecting the submucosa below the tumor burns and scars the submucosa, which tends to make the tumor-side edge rolled. Rolling makes it difficult for the endoscope tip to flip up the edge and apply upward traction.

\section{Successive short dissections make a safe and precise dissection line}

When starting to dissect the submucosa, hooking the submucosal tissue a slightly in the direction of the planned cut maintains a safe distance from both the muscularis and beneath the tumor. Then, press the pedal of the diathermy unit for a slightly less than one second, in order to make a short dissection. It is necessary to press it for the appropriate coagulation time, not extremely short, to avoid immediate bleeding from small blood vessels. Repeat multiple short dissections result in a safe and precise dissection line [Figure 5].

\section{An example of performing PCM}

A locally recurrent tumor in the rectosigmoid portion was found 6 years after multiple endoscopic piecemeal mucosal resections for cancer in an adenoma at another hospital [Figure 6]. Although the tumor had severe central submucosal fibrosis, PCM could deal with the fibrosis and achieved a complete resection. The resected specimen was $105 \mathrm{~mm}$ in diameter. Pathology revealed a high-grade adenoma with a negative margin.

\section{DISCUSSION}

It has been shown through retrospective studies and a prospective randomized control study that PCM facilitates colorectal ESD ${ }^{[3,5-7,10]}$. When comparing PCM to the conventional method, PCM has significant 


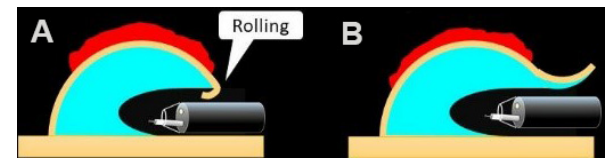

Figure 4. The advantage of a distant entrance. (A) When the initial mucosal incision as the entrance to the pocket is close to the tumor, the mucosal edge will be rolled. (B) A distant initial incision applies more upward traction and prevents rolling. The ideal distance is $14 \mathrm{~mm}$, the same as twice the diameter of the small-caliber-tip transparent hood orifice.

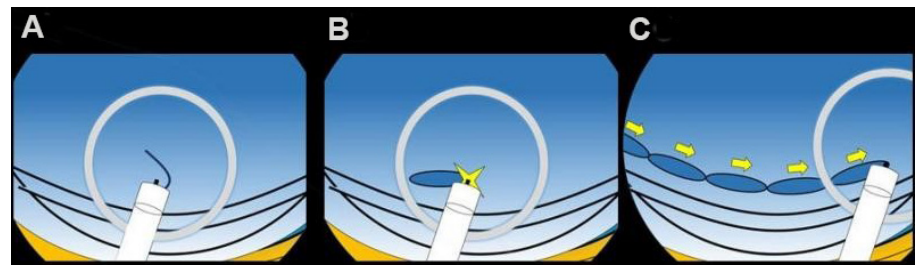

Figure 5. Successive short dissections. (A) Hooking the submucosa slightly in the direction of cut. (B) When pressing the pedal of the diathermy unit, the hooked submucosa is cut. (C) Performing successive multiple short dissections.

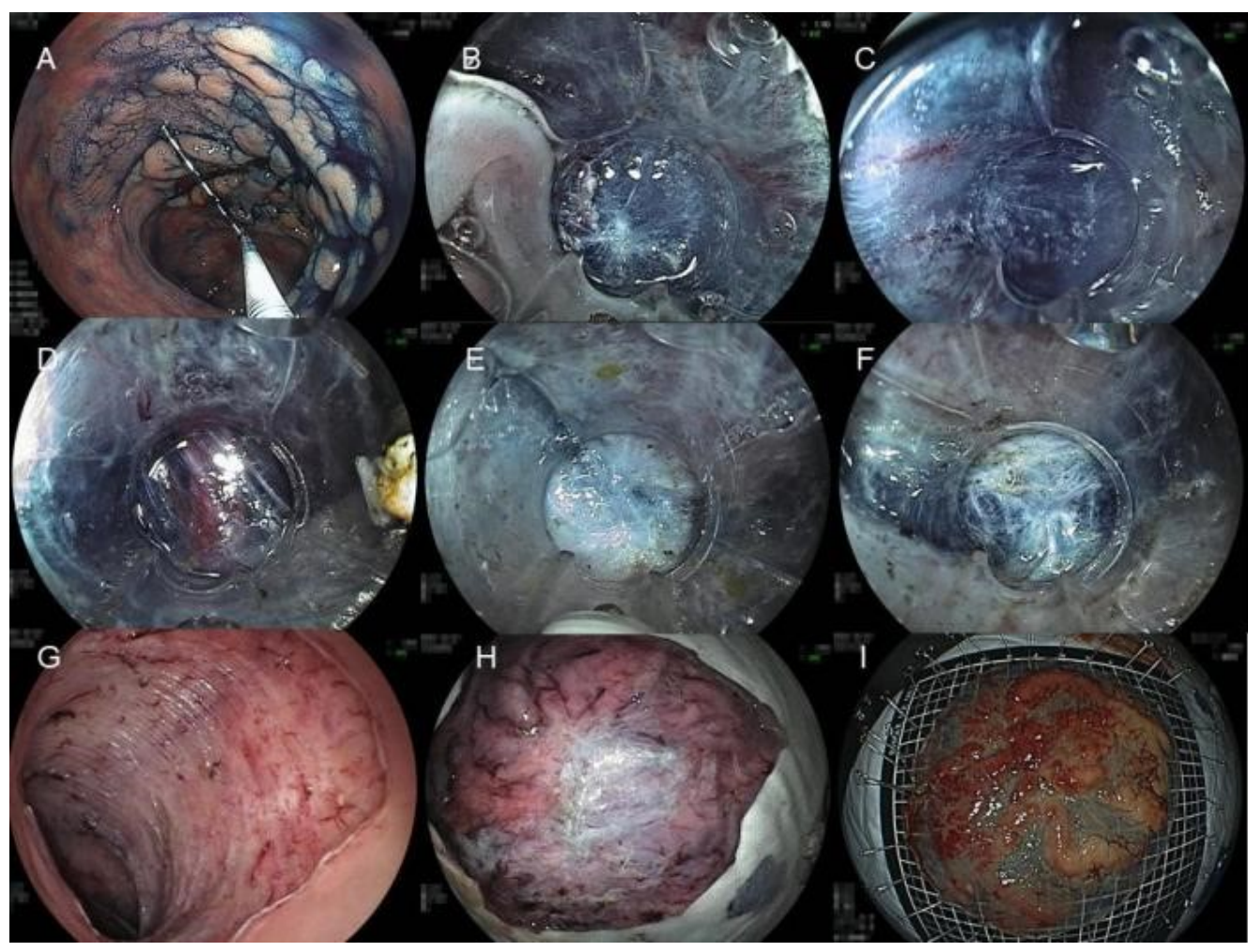

Figure 6. The pocket-creation method (PCM) of endoscopic submucosal dissection (ESD) for a locally recurrent tumor in the rectosigmoid portion. (A) A laterally spreading tumor approximately $8 \mathrm{~cm}$ in diameter. (B) The small-caliber-tip transparent (ST) hood entered the submucosal space below the tumor. Although using an ST hood with a small orifice, the entire view is secured through the transparent wall of the ST hood. A focal submucosal scar resulting from one of multiple biopsies performed at a local hospital was identified in the pocket. (C) The white surface of the muscularis was clearly identified distinguishing the blue submucosal layer in the pocket. (D) Perforating blood vessels were identified very clearly in the pocket so that they were precisely coagulated without immediate bleeding. (E) There was severe submucosal fibrosis due to previous multiple piecemeal endoscopic mucosal resections in the central area of the tumor. (F) After dissecting the fibrosis, the tip of the endoscope reached a less fibrotic area beyond the fibrosis. The submucosal pocket was subsequently opened. The ESD was completed in 211 min without any adverse events. (G) A mucosal defect after the ESD did not have any thermal damage. (H) The back of the ESD specimen showed a white severe fibrotic area. (I) The resected specimen and the tumor were $105 \mathrm{~mm} \times 87 \mathrm{~mm}$, and $82 \mathrm{~mm} \times 75 \mathrm{~mm}$ in size, respectively. Pathology revealed a high-grade adenoma with a negative margin. 
superiority to conventional ESD regarding en bloc resection, Ro resection, and dissection speed. Moreover, even if colonic ESDs using PCM were not performed by highly experienced endoscopists but rather by less experienced endoscopists, PCM resulted in better performance than conventional ESD ${ }^{[6]}$. When performing colorectal ESD with PCM, the ESD was completed using PCM without switching the strategy from PCM to other methods, PCM results in less switching compared to conventional method. When performing colorectal ESDs with PCM, it is not necessary for most endoscopists to consider changing their strategy of ESD according to each situation during the procedure.

A key feature of PCM is that both traction and countertraction are naturally produced by the tip of the ST hood in the submucosal pocket. The ST hood can stretch the submucosa at its orifice. This bidirectional traction by the ST hood is different from the traction by other traction devices which pull up not only the mucosa and the submucosa but also the muscularis unless countertraction such as insufflation or holding the muscularis with an endoscope tip is applied. The muscularis can be visually identified through the transparent wall of the ST hood and can be safely held with an edge of the ST hood. Therefore, endoscopists can focus on cutting the stretched submucosa without concern for damage to the pulled muscularis.

The usefulness of PCM was also shown for duodenal ESD ${ }^{[4]}$. PCM also facilitates extremely challenging ESD cases such as duodenal ESD. The duodenal submucosa is much thinner and rougher than other organs. Therefore, even if a viscous solution such as sodium hyaluronate is injected, the mucosal elevation cannot last as long as in other organs. In particular, solution injected into the submucosa in the flat area between folds may be dispersed quickly ${ }^{[1]}$. The minimal mucosal incision performed during PCM prevents leakage of the injected solution from the submucosa and the traction of ST hood stretches the thin submucosa in the pocket. Recently, our retrospective comparison study showed that PCM facilitates ESD of gastric tumors involving the pyloric ring ${ }^{[12]}$. Even the prominent ridge of the pyloric ring can be overcome with PCM during the submucosal dissection at the ring because undulation of the muscularis can be flattened using an ST-hood tip put on the tip of an endoscope in the limited-space pocket. This feature is also useful to overcome ridges of crescent folds in the colorectum ${ }^{[5]}$.

PCM is still evolving. Some additional techniques using saline immersion have been reported to facilitate performing $\mathrm{PCM}^{[13-16]}$. We know that some less experienced endoscopists may struggle to open the submucosal pocket at the end of PCM because the more opened the pocket is, the more unstable the endoscope tip becomes. Recently, we reported that a single reopenable clip facilitates opening the pocket ${ }^{[17]}$. Placing a clip to connect the specimen (after making the circumferential mucosal incision following creation of the pocket) to the opposite wall produces traction to stretch the remaining submucosa around the pocket. Single-clip traction is simpler and more economical than other dedicated traction devices ${ }^{[18]}$.

\section{CONCLUSION}

PCM is recommended as a standard strategy not only for colorectal ESD but also for upper gastrointestinal ESD. Although PCM has been established as an ESD strategy, it is expected that the use of traction techniques will make PCM easier to perform.

\section{DECLARATIONS}

\section{Authors' contributions}

Conception and design, drafting of the article, and final approval of the article: Hayashi Y, Miura Y

Drafting of the article, critical revision of the article for important intellectual content, and final approval of the article: Lefor AK 
Conception and design, drafting of the article, critical revision of the article for important intellectual content, and final approval of the article: Yamamoto $\mathrm{H}$

\section{Availability of data and materials \\ Not applicable.}

\section{Financial support and sponsorship}

None.

\section{Conflicts of interest}

Hironori Yamamoto has a patent for the small-caliber-tip transparent (ST) hood produced by the Fujifilm Corporation. The hood was used for ESD and shown in these pictures. He also has a consultant relationship with the Fujifilm Corporation and has received honoraria, grants, and royalties from the company. Other authors declared that there are no conflicts of interest.

\section{Ethical approval and consent to participate}

Not applicable.

\section{Consent for publication}

Not applicable.

\section{Copyright}

(c) The Author(s) 2022.

\section{REFERENCES}

1. Hayashi Y, Sunada K, Takahashi H, et al. Pocket-creation method of endoscopic submucosal dissection to achieve en bloc resection of giant colorectal subpedunculated neoplastic lesions. Endoscopy 2014;46 Suppl 1 UCTN:E421-2. DOI PubMed

2. Hayashi Y, Miura Y, Yamamoto H. Pocket-creation method for the safe, reliable, and efficient endoscopic submucosal dissection of colorectal lateral spreading tumors. Dig Endosc 2015;27:534-5. DOI PubMed

3. Kanamori A, Nakano M, Kondo M, et al. Clinical effectiveness of the pocket-creation method for colorectal endoscopic submucosal dissection. Endosc Int Open 2017;5:E1299-305. DOI PubMed PMC

4. Miura Y, Shinozaki S, Hayashi Y, Sakamoto H, Lefor AK, Yamamoto H. Duodenal endoscopic submucosal dissection is feasible using the pocket-creation method. Endoscopy 2017;49:8-14. DOI PubMed

5. Sakamoto H, Hayashi Y, Miura Y, et al. Pocket-creation method facilitates endoscopic submucosal dissection of colorectal laterally spreading tumors, non-granular type. Endosc Int Open 2017;5:E123-9. DOI PubMed PMC

6. Takezawa T, Hayashi Y, Shinozaki S, et al. The pocket-creation method facilitates colonic endoscopic submucosal dissection (with video). Gastrointest Endosc 2019;89:1045-53. DOI PubMed

7. Yamashina T, Nemoto D, Hayashi Y, et al. Prospective randomized trial comparing the pocket-creation method and conventional method of colorectal endoscopic submucosal dissection. Gastrointest Endosc 2020;92:368-79. DOI PubMed

8. Kitamura M, Miura Y, Shinozaki S, Yamamoto H. The pocket-creation method facilitates gastric endoscopic submucosal dissection and overcomes challenging situations. VideoGIE 2021;6:390-4. DOI PubMed PMC

9. Yamamoto H, Kawata H, Sunada K, et al. Successful en-bloc resection of large superficial tumors in the stomach and colon using sodium hyaluronate and small-caliber-tip transparent hood. Endoscopy 2003;35:690-4. DOI PubMed

10. Yamashina T, Hayashi Y, Fukuda H, et al. The pocket-creation method may facilitate endoscopic submucosal dissection of large colorectal sessile tumors. Endosc Int Open 2020;8:E1021-30. DOI PubMed PMC

11. Miura Y, Osawa H, Nomoto Y, Yamamoto H. Anatomical features of duodenal folds: a key feature to consider during endoscopic resection of duodenal neoplasms. VideoGIE 2021;6:529-32. DOI PubMed PMC

12. Kitamura M, Miura Y, Shinozaki S, et al. The pocket-creation method facilitates endoscopic submucosal dissection of gastric neoplasms involving the pyloric ring. Endosc Int Open 2021;9:E1062-9. DOI PubMed PMC

13. Harada H, Nakahara R, Murakami D, et al. Saline-pocket endoscopic submucosal dissection for superficial colorectal neoplasms: a randomized controlled trial (with video). Gastrointest Endosc 2019;90:278-87. DOI PubMed

14. Harada H, Murakami D, Suehiro S, et al. Water-pocket endoscopic submucosal dissection for superficial gastric neoplasms (with video). Gastrointest Endosc 2018;88:253-60. DOI PubMed

15. Yamamoto H, Hayashi Y, Despott EJ. The pocket-creation method for endoscopic submucosal dissection combined with salineimmersion: another potential option to overcome challenges in colorectal endoscopic submucosal dissection. Gastrointest Endosc 
2019;90:288-9. DOI PubMed

16. Despott EJ, Hirayama Y, Lazaridis N, et al. Saline immersion therapeutic endoscopy facilitated pocket-creation method for endoscopic submucosal dissection (with video). Gastrointest Endosc 2019;89:652-3. DOI PubMed

17. Nomura T, Hayashi Y, Lee RF, Takezawa T, Sunada K, Yamamoto H. Pocket-creation method using a new technique of single-clip traction for colorectal endoscopic submucosal dissection. Endoscopy 2020;52:E208-10. DOI PubMed

18. Nomura T, Hayashi Y, Morikawa T, et al. Colorectal endoscopic submucosal dissection using the pocket-creation method with single clip traction: a feasibility study. Endosc Int Open 2021;9:E653-8. DOI PubMed PMC 